\title{
Two-Dimensional Time Delay Measurement of a fast Scintillator using VLBI arrays
}

\section{Giuseppe Cimò*}

Joint Institute for VLBI in Europe

E-mail: cimo@jive.nl

Scintillation of flat spectrum radio sources provides a unique instrument to study the characteristics of the electron density distribution throughout the interstellar medium as well as to study the microarcsecond structure of these compact radio objects. A new approach, using VLBI techniques, to measure the distance of the scattering screen in the interstellar medium will be described. The aim is to measure the time delay, caused by the scintillation pattern, between the different array elements. Results of recent EVN observations will be presented.

The 9th European VLBI Network Symposium on The role of VLBI in the Golden Age for Radio Astronomy and EVN Users Meeting

September 23-26, 2008

Bologna, Italy

${ }^{*}$ Speaker. 


\section{Introduction}

IntraDay Variable sources (IDV) are flat spectrum extragalactic synchrotron sources, often associated with optical quasars, BL Lacs or radio galaxies, that show flux density variability on time scales shorter than a day (Kedziora-Chudczer et al. 1997). They are typically unresolved with the longest VLBI baselines at $\mathrm{cm}$-waves. These sources show intrinsic variations on much longer time scales (months) that sometimes are associated with milliarcsecond structure that evolves on time scales of years (Krichbaum et al. 1997), but the easiest way to understand the intraday variability is as a propagation effect, similar to that in low frequency variables, due to scintillation in an interstellar screen of ionized gas (Rickett et al. 2001 and references therein). In this paradigm, the refractive screen acts like the agitated surface of a swimming pool, that bends sunlight to cause an irregular pattern of light and dark bands on the bottom of the pool. In the astronomical case, the Earth moves through an immense pattern of light and dark bands, which causes the variability of the IDV sources. This theory was confirmed by Dennett-Thorpe \& de Bruyn (2002) who monitored flux density variations in J1819+3845 at the VLA and WSRT simultaneously. The light curves from the two telescopes show nearly perfect correlation, but with a time delay of a few minutes, corresponding to the separation of the two telescopes in their motion through the refraction pattern. Bignall et al. (2003) observed a similar pattern in the source PKS 1257-326 using the VLA and the ATCA.

\section{The original idea: proposed technique}

Rather than using just two observatories, which gives just one number for the time delay, we used the EVN, so as to get time delays between every pair of antennas and to determine the two dimensional velocity vector of the Earth relative to the refraction pattern. With the magnitude of this vector, $v$, it is possible to determine the distance to the screen, in the case where the source is in the weak scattering limit (Rickett et al. 2001, Bignall et al. 2003). This is because the characteristic spatial scale of the pattern is set by the Fresnel zone size, $r_{F}$,

$$
r_{F}=\sqrt{\frac{\lambda L}{2 \pi}}
$$

with $L$ the distance to the screen. In this weak scattering limit the time scale of the variability is $t_{w}$,

$$
t_{w}=\frac{r_{F}}{v}
$$

with $v$ the velocity of the Earth relative to the screen.

The selected object for such experiment was the fast scintillator J1819+3845, which has been knows as the most extreme IDV source (600\% variability within a few hours). Due to the annual modulation of the time scales, this source showed its fastest variations between February and April (Dennett-Thorpe \& de Bruyn, 2003). The variability has been observed continuously for more than 8 years.

Such measurements can give us information about the scattering screen to the line of sight of $\mathrm{J} 1819+3845$, providing a new accurate and immediate method to determine the distance of the interstellar ionized compact structures. 


\section{Observations}

To track the varying flux density of a typical IDV requires measurements with precision of a few mJy on time scales of a few minutes. Observing with the EVN, we make use of the fact that the source remains unresolved on all baselines, so the variability as seen by each antenna shows up just like a gain variation. If we assume a constant flux density, then a standard closure-amplitude solution for the antenna gains and phases results in a profile of the total intensity as seen by each antenna. Frequent observation of the calibration source J1821+3945 helps to separate the true antenna gain variations from the program source variation, but even without that, the cross-correlation of the antenna gain versus time between different antennas should show a peak at the delay caused by the scintillation pattern. These delays should be consistent with a single velocity vector caused by the relative motion of the Earth and the screen.

We observed 12 hours at the EVN at $4.8 \mathrm{GHz}$ in order to measure the time delay in the weak scattering regime. The idea was to observe a few maxima and minima in the variability pattern and to better constrain the observed delays. The observations were carried out on March 12, 2008. The telescopes involved were: Westerbork, Effelsberg, Cambridge, Jodrell Bank, Onsala, Noto, Medicina, Torun, Urumqi and Shangai. Unfortunately bad weather conditions affected most of the antennas. In particular the 76-meter antenna in Jodrell Bank missed all the experiment due to high wind. Furthermore the station in Shangai had severe problems with the recording system.

\section{The 'Unexpected' Result}

From the analysis of the amplitudes of the single EVN baselines, but mainly from the Westerbork synthesis data, we observed a surprising lack of any variability of J1819+3845!

Episodic variability is a common phenomenon in flat spectrum radio sources. In some cases, longterm (within months or years) changes in the IDV pattern can be due to source-intrinsic effect, such as the emission of new components quenching the scintillation. A source scintillates as long as its angular size is smaller than the Fresnel scale in our Galaxy. The scintillations would stop once the component expands exceeding the Fresnel size. The disappearance of the extreme scintillations on J1819+3845 could be due to variations in the source structure at $\mu$-arcsecond scale. A new component, which expands as it evolves would lead to episodic scintillations. On the other hand, evidences for a new component such as strong changes in flux density have not been observed. Finally, assuming a new component emitted from the core has emerged, we used the EVN data to see if this component is resolved at milliarcsecond scale a year after the last recorded variability. However, the source appears unresolved on all EVN baselines.

A change in the scattering material along the line of sight to J1819+3845 is another possible (and maybe more plausible) explanation. We can assume that the screen has simply moved away, and therefore we can immediately put some constraints on the size of the scattering material. Assuming a typical velocity of the cloud of about $20 \mathrm{~km} / \mathrm{s}$, this will result in $210^{-5} \mathrm{pc} / \mathrm{yr}$. Therefore, the screen moved of $4.2 \mathrm{AU}$ in one year. Since we do not know when the scintillation started, we cannot define 
the linear size of the screen. As a limit, we can evaluate a size $>34$ AU. More detailed analysis (Cimò et al. in prep., de Bruyn et al. in prep.) is in progress.

\section{Conclusions}

The European VLBI Network has been used to repeat the measurement of time delays using all the baselines of the VLBI array. The unexpected outcome of this experiment has shown that the extreme variability of $\mathrm{J} 1819+3845$ has stopped.

Taking into account previous IDV measurments of this quasar, it has been possible to put a lower limit of $34 \mathrm{AU}$ to the size of the cloud responsible of the previously observed extreme variations. Due to the lack of variability, it has been possible to image the source. After a year from the last observed scintillation, the source is unresolved on VLBI scale.

Local ISM changes (the screen moved away) can explain the end of the extreme scintillation of $\mathrm{J} 1819+3845$. Theoretical studies are in progress to constrain the screen characteristics.

\section{References}

[1] H. E. Bignall, D. 1. Jauncey, J. E. J. Lovell, A. K. Tzioumis, et al. 2003, Rapid Variability and Annual Cycles in the Characteristic Timescale of the Scintillating Source PKS 1257-326, Ap. J. 585 (653).

[2] J. Dennett-Thorpe \& A. G. de Bruyn, 2002, Interstellar scintillation as the origin of the rapid radio variability of the quasar J1819+3845, Nature 415 (57).

[3] J. Dennett-Thorpe \& de A. G. Bruyn, 2003, Annual modulation in the scattering of J1819+3845: Peculiar plasma velocity and anisotropy, A\&A 404 (113).

[4] L. Kedziora-Chudczer, D. L. Jauncey, M. H. Wieringa, M. A. Walker, et al. 1997, PKS 0405-385: The Smallest Radio Quasar?, Ap. J. 490 (L9).

[5] T. P. Krichbaum, D. A. Graham, A. Greve, J. E. Wink, et al. 1997, 215 GHz VLBI observations of bright Active Galactic Nuclei, A\&A 323 (17).

[6] B. J. Rickett, A. Witzel, A. Kraus, T. P. Krichbaum \& S. J. Qian, 2001, Annual Modulation in the Intraday Variability of Quasar 0917+624 due to Interstellar Scintillation, Ap. J. 550 (L11). 